\title{
Fluid Modeling of an ELMing H-mode and a RMP H-mode
}

\author{
S. Mordijck ${ }^{a}$ E.A. Unterberg ${ }^{b}$ R.A. Moyer ${ }^{a}$ T.E. Evans ${ }^{c}$ \\ L.W. Owen ${ }^{d}$ M. Groth ${ }^{\text {e }}$ T.H. Osborne ${ }^{c}$ T.W. Petrie ${ }^{c}$ \\ J.G. Watkins ${ }^{\mathrm{f}}$ D. Reiter ${ }^{\mathrm{g}}$ X. Bonnin ${ }^{\mathrm{h}}$ D. Coster $^{\mathrm{i}}$ J. Canik $^{\mathrm{d}}$ \\ R. Maingi ${ }^{\mathrm{d}}$ \\ ${ }^{a}$ University of California San Diego, La Jolla, California, USA \\ ${ }^{\mathrm{b}}$ Oak Ridge Institute of Science Education, Oak Ridge, Tennessee, USA \\ ${ }^{\mathrm{c}}$ General Atomics, San Diego, California 92186-5608, USA \\ ${ }^{\mathrm{d}}$ Oak Ridge National Laboratory, Oak Ridge, Tennessee, USA \\ ${ }^{\mathrm{e}}$ Lawrence Livermore National Laboratory, Livermore, California, USA \\ ${ }^{\mathrm{f}}$ Sandia National Laboratories, Albuquerque, New Mexico, USA \\ ${ }^{\mathrm{g}}$ Forschungszentrum Juelich, Juelich, Germany \\ ${ }^{\mathrm{h}}$ Laboratoire d'Ingénierie des Matériaux et des Hautes Pressions - CNRS, Paris, \\ France \\ ${ }^{\mathrm{i}}$ Max-Planck Institut für Plasmaphysik, Garching, German
}

\begin{abstract}
In this paper we investigate the role of wall conditions during the density pumpout observed in Resonant Magnetic Perturbation (RMP) experiments. We use a 2D fluid code, coupled to a neutral Monte-Carlo code (SOLPS5) to model a reference
\end{abstract}


H-mode. Next we modify either the recycling at the target plates or the pumping efficiency at the pump entrance. This causes a change in upstream profiles, that alone cannot account for the density pump-out observed in the RMP H-mode. Therefore, the radial transport must be altered to match the RMP H-mode. By comparing the transport model for the ELMing H-mode and the RMP H-mode, we show that inside the separatrix an increase in transport is necessary to model the RMP H-mode, which is due to the applied stochastic field.

Key words:

\section{Introduction}

Edge Localized Modes (ELMs) are predicted to have a critical heat load, that could lead to serious erosion in ITER (1) - (2). Reducing the impulsive power loading at the divertor target plates due to ELMs is therefore critical to the operation of ITER. Recently it has been shown that Resonant Magnetic Perturbations (RMP) successfully suppress ELMs (3), by lowering the pressure gradient below the peeling-ballooning stability limit (4). The density pumpout observed in RMP experiments is the prime factor for lowering the pressure gradient.

In this paper we investigate the effects of wall conditions and pumping with respect to the density pump-out observed during RMP experiments on DIIID. When the I-coil is energized with $n=3$ magnetic perturbations at low pedestal collisionality, density pump-out occurs, and as a result the divertor conditions change (5), i.e., a lower density strike-point, and a broadening of the

Email address: smordijc@ucsd.edu (S. Mordijck). 
density profile on the divertor target. Thus, the amount of plasma wetted area increases, which alters recycling at the target plates, and can lower the pedestal density (6). In addition experimental and modeled heat flux patterns show strike-point splitting (7). Since the location of the strike-point determines the pumping efficiency, e.g., moving the strike-point closer to the pump entrance increases the pumping efficiency (8), which affects the global particle balance. Therefore, we will investigate whether the density pump-out is primarily due to changes in recycling and pumping efficiency, or if transport enhancement is required for density pump-out in RMP H-mode.

To distinguish between the effects of strike-point splitting and transport, we use a 2D fluid code coupled to a Monte Carlo neutral code (9). In the last decade, several parallel versions of this code have been developed and in this paper SOLPS5 (10) is employed. Starting from a reference ELMing H-mode the transport coefficients are computed, and kept fixed for the remainder of the numerical experiment. In order to validate the simulated effects of strikepoint splitting, we scan a predefined range of either the recycling coefficients for the target plates or the pumping efficiency at the plenum of the cryo-pump. For all numerical results, we observe that the midplane density profile drops and the shape of the pedestal remains constant. In contrast, the experimental results show a change in the pedestal shape. Therefore, a change in transport is required to induce this change. Transport inside the separatrix has to increase in the area of the transport barrier to simulate the huge drop in density of the pedestal, while keeping a nearly similar density in the scrape-off-layer (SOL).

The main results of this paper are how the divertor conditions affect midplane profiles. Also, we show that reduced recycling at the target plates and better pumping efficiency does not solely account for the density pump-out observed 
during RMP experiments.

\section{Method}

This section describes the relevant experimental data and the modeling approach. First, we introduce the experimental setup and most important results. Next, we describe the process followed for the numerical experiments.

To distinguish between a standard H-mode, which will serve as reference, and an RMP H-mode, two experiments are carefully selected. The RMP H-mode has exactly the same setup as the reference H-mode, except for the activation of the I-coil at $2000 \mathrm{~ms}$. The two discharges evolve differently subsequent to the I-coil turn-on. Thus, experimental data is identical for both shots upto 2000ms, see Figure 1. Comparing both experiments after switching on the I-coil, shows differences on a short time scale,i.e., $50 \mathrm{~ms}$, and on a longer scale, i.e., $300 \mathrm{~ms}$. Three rapid ELMs are observed in the divertor of the RMP H-mode, within the first $50 \mathrm{~ms}$ after switching on the I-coil, together with a pressure increase under the baffle. Additionally, a sudden drop in the line averaged density and an increase in $\mathrm{D} \alpha$ light at both target plates is observed. The line averaged density continues decreasing for an extended period of time, upto $300 \mathrm{~ms}$ after RMP initialization. The pressure under the baffle decreases in a similar fashion, similar to the $\mathrm{D} \alpha$ light at the outer strike-point. On the otherhand, the $\mathrm{D} \alpha$ emmission at the inner strike-point increases. The changed $\mathrm{D} \alpha$ and pressure imply a change in the recycling coefficients and pumping efficiency which needs to be modeled.

To understand the effects of recycling at the target plates and the pumping 
efficiency, we select a time slice to model, where most variables are relatively stable in the ELMing H-mode (whith exception of the ELMs) and the RMP H-mode, namely at $2675 \mathrm{~ms}$, see Figure 1. This time slice is located between two ELMs for the ELMing H-mode. Also, the transient behavior caused by the ELMs is not modeled. Next, we construct a grid, based on the equilibrium data of the ELMing H-mode. To derive the transport model, we apply the same techniques discussed in (11). At the core boundary, fixed density and temperatures are chosen as boundary conditions based on experimental data. The SOL and Private Flux Region (PFR) boundary conditions are characterized by short decay lengths, allowing poloidal variation of the effective values. The Bohm-Chodura sheath boundary condition is used at the target plates. Boundary conditions for EIRENE are the recycling coefficients, which are set to 1 everywhere, with exception of the target plates where 0.97 is used. The pumping efficiency is set at the opening under the baffle at a value of $30 \%$. The transport model is varied until the modeled profiles are comparable to the experimental profiles. The final transport model is fixed for the subsequent numerical experiments.

Our numerical experiment starts by reducing the recycling in the divertor and we examine the effect on the upstream profiles by comparing the numerical profiles to the experimental data from the ELMing H-mode. Then, in a similar fashion the pumping efficiency is independently altered and profiles are again compared with the ELMing H-mode. Finally, we model the RMP H-mode by changing the transport model to fit the changed pedestal structure. A comparison of the transport model from the ELMing H-mode with the transport model of the RMP H-mode gives an indication of the effect of the applied magnetic perturbations on the transport. 
All simulations are performed without drifts and impurities. To simulate the influence of Carbon, an artificial sputtering coefficient is set at 2\% Carbon sputtering over the whole vessel. Also all the results are obtained with a radial velocity pinch, at $v=4 \mathrm{~m} / \mathrm{s}$. Due to lack of experimental data to ascertain how $v$ might be changing, this number is kept fixed. Also, when matching the ELMing H-mode and the RMP H-mode, the thermal transport is adapted to match temperature profiles.

\section{Results and Discussion}

In this section, we first discuss the resulting transport model for the ELMing H-mode. Next, the results for the numerical experiments are detailed. Finally, we discuss the comparison of the two transport models.

\subsection{Transport model ELMing H-mode}

The transport model is derived by matching the experimental midplane profiles. Figure 2 shows the measured electron density with experimental errorbars. The solid curve is the fitted SOLPS5 data and in figure 6 the relative transport coefficients are shown. The values for the particle diffusivity, $D_{n}$, and the thermal diffusivity $\kappa_{e}$ are of the same magnitude as Gulejová et. al. (11) and comparable to commonly used estimates. Note that only one probe measurement is available near the outer strike-point. The peak values given by our similution are within a factor of 2 to 3 of the probe measurements. 


\subsection{Numerical experiments}

After fixing the transport model, and changing the recycling coefficient at the target plates from 0.97 to $0.95,0.93$ and 0.91 , a reduction in the divertor density profile is observed, see Figure 3. Moreover, this reduction also manifests itself upstream in the density profile. The whole density profile, drops with reduced recycling in the divertor. This can be explained by an increased number of plasma particles that are now captured in the target plates, instead of being recycled as neutrals, which can re-ionize and fuel the core. However, we observe that only changing the recycling at the target plates is not enough to reach RMP H-mode conditions:

- The peak density only drops by a factor 1.5 in the numerical experiments compared to a factor 3 ,

- The midplane density profile change is not substantial enough and falls within the errorbars of the ELMing H-mode.

Changing the pumping efficiency from 0\%-100\% shows again the importance of neutral fueling. The numerical runs can become unstable with the chosen boundary core boundary conditions if too few neutrals are pumped from the divertor area. Similarly, pumping more particles, not only reduces the density distribution in the divertor, but also reduces the profiles at the midplane, see Figure 4. The reduction is more substantial for increased pumping efficiency when compared to changing the recycling at the target plates:

- In the divertor a drop of a factor 4 is observed when going from $30 \%$ pumping to $90 \%$ pumping,

- In the divertor an increase is observed of a factor 2, when going from $30 \%$ 
pumping to only $10 \%$ pumping,

- The drop in density in the midplane profiles is insufficient and reaches a threshold.

Comparing these reductions to experimental data, shows that we can not achieve RMP H-mode conditions by only changing the wall conditions in the divertor. The pump, which has the biggest effect, needs to increase its efficiency, wherease experimental data shows a decrease of particles in the pump. When comparing particle balances for these two experiments, during I-coil operation, the pump in the RMP H-mode pumps three times less particles than the ELMing H-mode, i.e., 9.5 versus 30.9 torr liters.

Moreover, changing the recycling and the pumping efficiency does not alter the pedestal shape, which remains steep, whereas the RMPs modify the pedestal shape. Therefore, the RMP H-mode necessitates a new transport model.

\subsection{Transport Model RMP H-mode}

Figure 5 shows the results for the RMP H-mode. The solid line represents the modeled data and the dots the experimental. To achieve these results, the altered transport model is shown in Figure 6. In the same figure, the transport model for the ELMing H-mode is also displayed.

Between $\psi_{n}$ of 0.8 and 0.9 both diffusion coefficients are comparable. From $\psi_{n}$ 0.9 to 1, the transport model for the ELMing H-mode exhibits a dip. This dip is needed to obtain the steep density profile just inside the seperatrix, whereas the density profile for the RMP H-mode is much more gradual and does not require this reduction in diffusion. At the extremes of the SOL, both curves 
cross. In this region, the diffusion coefficient is dominated by the neutrals and the changes to the diffusion coefficient are less sensitive than inside the seperatrix.

\section{Conclusion}

In this paper, we derived a transport model for an ELMing H-mode for the DIII-D tokamak. Next, we showed that reduced recycling in the divertor, not only reduces the density distribution in the divertor, but also in the upstream profiles. Futhermore, we showed that increasing pumping efficiency has a similar, but more pronouced, effect. As a result we showed that these effects are not enough to account for the changes observed in the RMP H-mode. Therefore, the transport model was changed for the RMP H-mode. Comparing the transport model for the RMP H-mode with the transport model for the ELMing H-mode shows enhanced transport inside the separatrix. This enhanced transport is the result of the penetration of stochastic field lines.

Future research is needed to understand whether RMP is amplified or screened. First, we need to calculate the difference of the diffusion coefficient of the RMP H-mode and the ELMing H-mode. Then comparing this difference with the stochastic vacuum field line diffusion, can show amplification or screening.

\section{Acknowledgments}

Work supported by the U.S. Department of Energy under DE-FG02-04ER54758, DE-FC02-04ER54698, W-7405-ENG-48, DE-AC05-00OR22725, DE-AC05-76OR00033, 
and DE-AC04-94AL85000. Special thanks to Pieter Peers.

\section{References}

[1] I. P. B. Editors, Nucl. Fusion 39 (1999) 2137.

[2] K. Ikeda, Nucl. Fusion 47 (2007) S1.

[3] T. E. et. al., Phys. Plasmas 13 (2006) 0256121.

[4] P. S. et. al., Phys. Plasmas 9 (2002) 2037-2043.

[5] J. W. et. al., Journal Nucl. Mat. 363-365 (2007) 708-712.

[6] R. M. et. al., Nucl. Fusion 36 (1996) 245-253.

[7] I. J. et. al., Nucl. Fusion 48 (2008) 045009.

[8] R. M. et. al., Nucl. Fusion 39 (1999) 1187-1192.

[9] D. Reiter, J. Nucl. Mat. 196-198 (1992) 80.

[10] R. S. et. al., Contrib. Plasma Phys. 40 (2000) 328-333.

[11] B. G. et. al., J. Nucl. Mat. 363-365 (2007) 1037. 


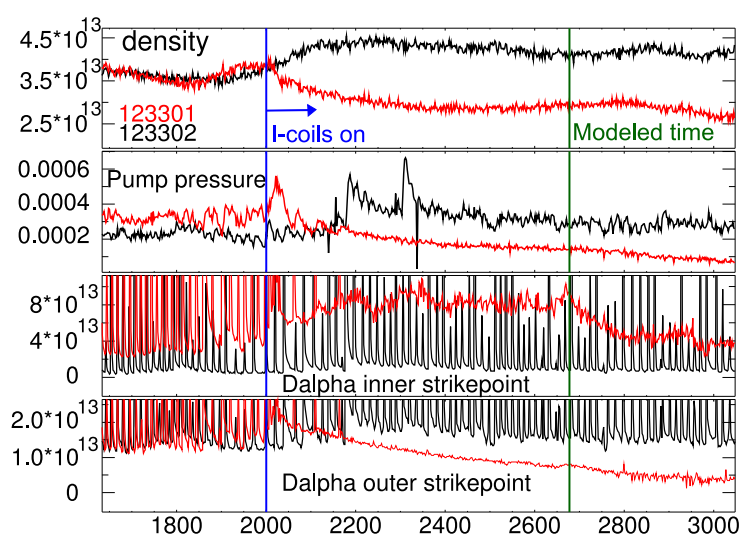

Fig. 1. Experimental results for the ELMing H-mode and the RMP H-mode

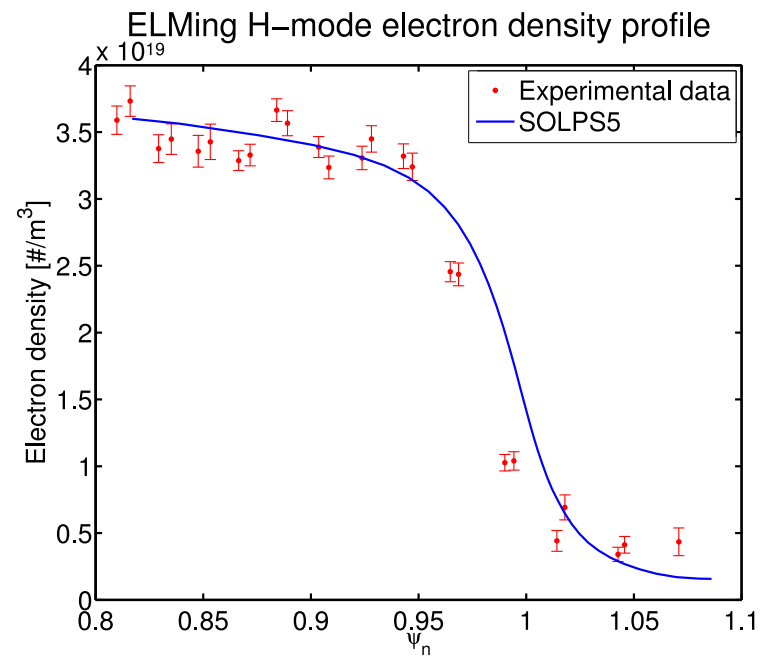

Fig. 2. Experimental and SOLPS5 data for the ELMing H-mode

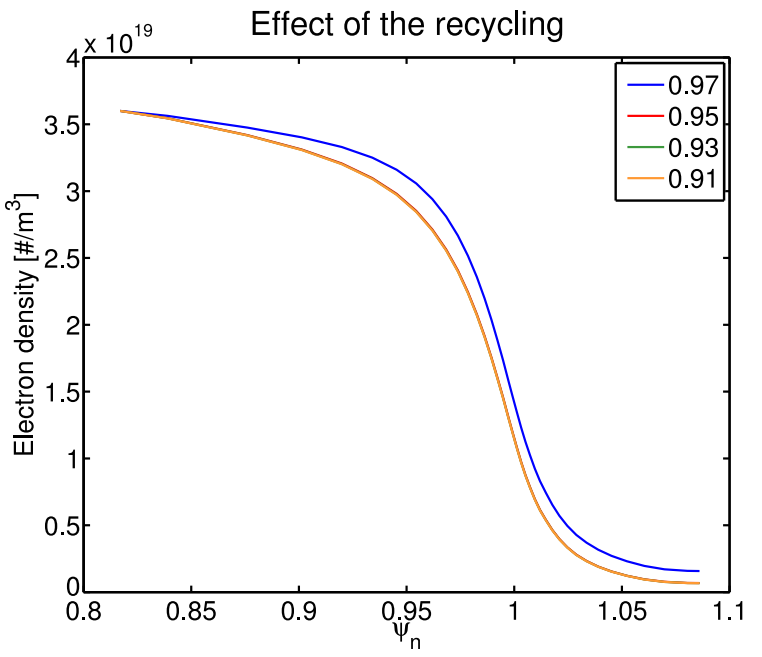

Fig. 3. Effect of changed recycling at the target plates 


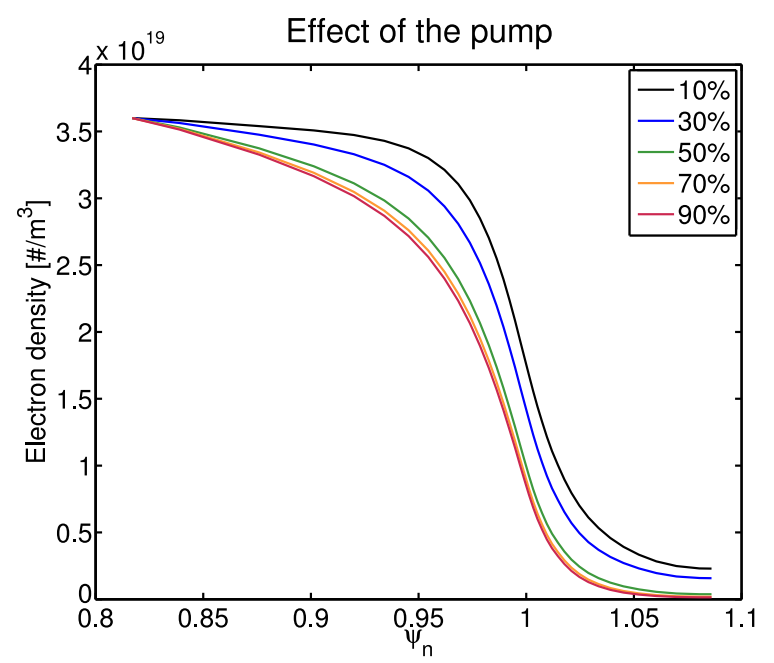

Fig. 4. Effect of changed pumping efficiency

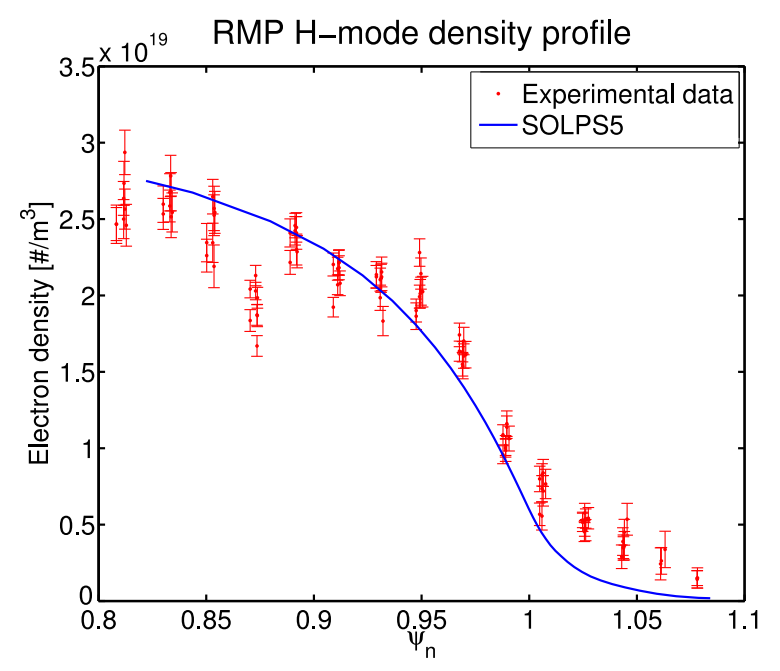

Fig. 5. Experimental and SOLPS5 data for the RMP H-mode 


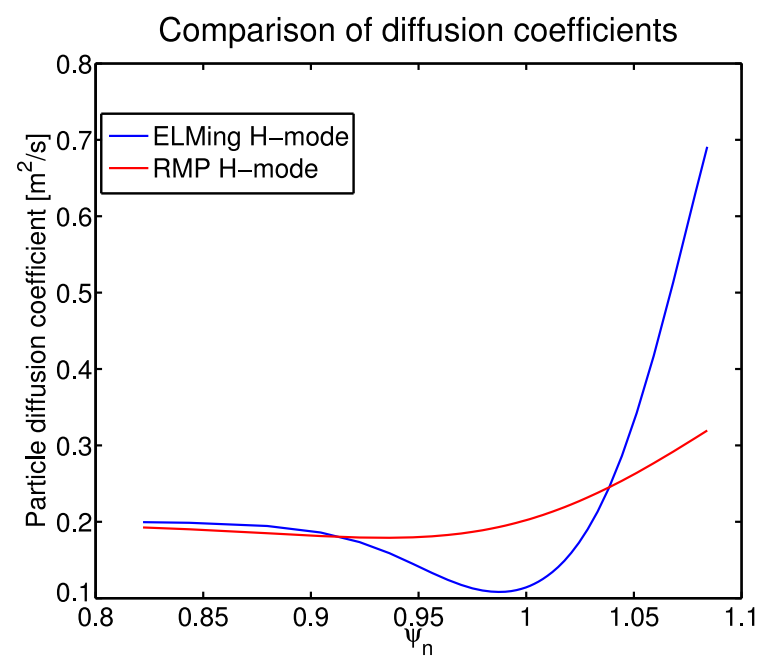

Fig. 6. Diffusion coefficient comparison between ELMing H-mode and RMP H-mode 